Revue internationale P.M.E.

Économie et gestion de la petite et moyenne entreprise

\title{
Le concept de contrôle du destin et son influence sur le comportement entrepreneurial
}

\section{Egbert McGraw et Yves Robichaud}

Volume 8, numéro 2, 1995

URI : https://id.erudit.org/iderudit/1008350ar

DOI : https://doi.org/10.7202/1008350ar

Aller au sommaire du numéro

Éditeur(s)

Presses de l’Université du Québec

ISSN

0776-5436 (imprimé)

1918-9699 (numérique)

Découvrir la revue

Citer cet article

McGraw, E. \& Robichaud, Y. (1995). Le concept de contrôle du destin et son influence sur le comportement entrepreneurial. Revue internationale P.M.E., 8(2), 79-97. https://doi.org/10.7202/1008350ar

\section{Résumé de l'article}

Le contrôle du destin est décrit dans la littérature comme une caractéristique ayant une grande influence sur le comportement entrepreneurial. Dans cette perspective, le présent article examine les relations entre les trois dimensions suivantes: le contrôle du destin, les pratiques de gestion et certaines caractéristiques personnelles d'un échantillon d'entrepreneurs francophones du Nouveau-Brunswick et du nord-est de l'Ontario. Les résultats ont démontré que certaines variables telles que la taille de l'entreprise, le type, ainsi que les années d'études, ont un effet sur les pratiques de gestion. En effet, plus le niveau de scolarisation est élevé, plus on pratique des activités de gestion. D’autres variables mesurant les caractéristiques personnelles des dirigeants se sont aussi avérées significatives. La variable contrôle du destin fut reliée à une plus grande utilisation des pratiques de gestion par les entrepreneurs. 


\title{
Le concept de contrôle du destin et son influence sur le comportement entrepreneurial ${ }^{1}$
}

\author{
Egbert McGRAW* \\ Université de Moncton \\ Yves ROBICHAUD** \\ Université Laurentienne
}

MOTS CLÉS

\section{Entrepreneurship - Contrôle du destin \\ Pratiques de gestion - Entrepreneurs}

\begin{abstract}
RÉSUMÉ
Le contrôle du destin est décrit dans la littérature comme une caractéristique ayant une grande influence sur le comportement entrepreneurial. Dans cette perspective, le présent article examine les relations entre les trois dimensions suivantes: le contrôle du destin, les pratiques de gestion et certaines caractéristiques personnelles d'un échantillon d'entrepreneurs francophones
\end{abstract}

1. Cette recherche fut rendue possible grâce à une subvention de l'Association des universités partiellement ou entièrement de langue française - Université des réseaux d'expression francaise (AUPELF-UREF). Cette recherche a été présentée au $11^{\mathrm{e}}$ colloque du Conseil canadien de la PME et de l'entrepreneuriat à Winnipeg en 1994.

* Egbert McGraw, D.S.S., B.Sc. M.B.A., Ph.D. F.C.G.A., est professeur au Département de comptabilité de l'Université de Moncton. Avant de joindre les rangs à la Faculté d'administration, $\mathrm{M}$. McGraw a travaillé dans le financement de la PME à la Banque Fédérale de Développement et a été directeur de développement immobilier pour une entreprise spécialisée dans le développement et la gestion de centres commerciaux. Ses domaines de recherche portent sur l'entrepreneurship, la comptabilité sociale ainsi que l'impact économique des normes comptables. Adresse : Université de Moncton, Faculté d'administration, Moncton, Nouveau-Brunswick, Canada, E1A 3E9.

** Yves Robichaud enseigne au Département de comptabilité de l'Université Laurentienne de Sudbury en Ontario. Il détient un baccalauréat en administration des affaires et une maîtrise en administration publique de l'Université de Moncton et est membre de l'Association des comptables généraux licenciés de l'Ontario. Il poursuit actuellement un doctorat en sciences de la gestion de l'Institut d'administration des entreprises de l'Université de droit, d'économie et des sciences d'Aix-Marseille. Adresse: École de commerce et d'administration, Université Laurentienne, Sudbury, Ontario, P3E 2C6 
du Nouveau-Brunswick et du nord-est de l'Ontario. Les résultats ont démontré que certaines variables telles que la taille de l'entreprise, le type, ainsi que les années d'études, ont un effet sur les pratiques de gestion. En effet, plus le niveau de scolarisation est élevé, plus on pratique des activités de gestion. $D$ 'autres variables mesurant les caractéristiques personnelles des dirigeants se sont aussi avérées significatives. La variable contrôle du destin fut reliée à une plus grande utilisation des pratiques de gestion par les entrepreneurs.

\begin{abstract}
The literature describes locus of control as a characteristic having a strong influence on entrepreneurial behaviour. This article builds on this perspective by examining the relationships among the following three dimensions: locus of control, management techniques and personal characteristics of a sample of francophone entrepreneurs from New Brunswick and Northeastern Ontario. The survey results indicated that the size of the firm, the type and level of education influence management techniques. Specifically, higher levels of education were associated with increasing levels of activities related to the management techniques. Other personal variables were also found to be significant. Locus of control was positively associated with the management techniques used by these entrepreneurs.
\end{abstract}

\title{
RESUMEN
}

En lo que hay escrito sobre el tema se describe el locus de control como una característica que ejerce una fuerte influencia sobre el comportamiento empresarial. Este artículo ahonda en esta perspectiva al examinar las relaciones existentes entre las tres dimensiones siguientes: el control del destino, las técnicas de gestión y las características personales de una muestra de empresarios francoparlantes de las provincias de New Brunswick y del noroeste de Ontario. Los resultados de la encuesta indicaron que tanto el tamaño de la empresa como el tipo y nivel de educación afectan a las técnicas de gestión. Así, cuanto más alto es el nivel de educación máyor es la frecuencia con la que se practican actividades relacionadas con las técnicas de gestión. Las conclusiones revelan además la importancia significativa de otras variables relativas a la personalidad. Se considera positivo el efecto que la variable locus de control ejerce sobre las técnicas de gestión al uso de los empresarios encuestados.

\section{Introduction}

Le domaine de la recherche sur l'entrepreneurship est en pleine évolution, bien que plusieurs questions, telle la définition d'un entrepreneur, ne font toujours pas l'unanimité chez les chercheurs. Malgré cette situation, ces derniers s'entendent néanmoins sur un certain nombre de traits de personnalité associés au fait d'être entrepreneur. Les définitions utilisées dans la littérature pour décrire ces traits varient d'un chercheur à l'autre, mais les regroupements suivants sont généralement acceptés : créativité et innovation, besoin d'indépendance 
et d'autonomie, besoin d'accomplissement, tendance à la prise de risques modérés et contrôle du destin.

Parmi ces traits, le contrôle du destin, ou l'internalité, a été décrit à plusieurs reprises dans la littérature comme une caractéristique de personnalité cruciale en entrepreneurship (Borland, 1975 ; Shapero, 1975; Kets de Vries, 1977 ; Pandey et Tewary, 1979; Miller et al., 1982; Miller, 1983; Miller et Toulouse, 1986). Plus particulièrement, il semble être un élément prometteur pour expliquer une plus grande utilisation des pratiques de gestion puisque, selon certains auteurs, ce concept influence la recherche et l'utilisation d'information (Seeman et Evans, 1962 ; Davis et Phares, 1967 ; Seeman, 1967 ; Phares, 1968 ; Gordon et Miller, 1978 ; Hendrix, 1985).

La dimension contrôle du destin a été développée par Rotter (1966) dans le cadre de sa théorie de l'apprentissage social par le renforcement. Le contrôle du destin décrit un concept de soi où l'individu attribue sa performance à sa propre responsabilité plutôt qu'à celle des autres et où il croit qu'il peut modifier son environnement plutôt que de se croire soumis à la chance et au destin, ce qui est le cas du concept de soi opposé : l'externalité (Cachon, 1992).

Cette perception de leur capacité ou de leur incapacité d'influencer les événements entourant leur vie se manifeste par des comportements entrepreneuriaux différents. Ainsi, l'individu qui présente un concept de soi orienté vers l'internalité, donc qui se croit en mesure d'influencer les événements qui se produisent, aura tendance à utiliser tout ce qui est à sa disposition pour influencer ces événements. De l'autre côté, l'individu qui tend vers l'externalité, aura tendance à demeurer passif devant une situation donnée, puisqu'il relie les résultats d'une action à la chance ou à d'autres facteurs hors de son contrôle.

C'est dans cette perspective que cette étude tentera d'établir une relation entre trois dimensions de l'entreprise dont les caractéristiques de l'entreprise, les caractéristiques de l'entrepreneur, ainsi que les pratiques de gestion utilisées. La figure 1 fait ressortir cette relation en plus d'identifier les variables reliées aux profils de l'entrepreneur et de l'entreprise, ainsi que les pratiques de gestion regroupées autour des fonctions de l'entreprise. La littérature qui supporte la relation entre ces trois dimensions est présentée dans les sections deux et trois intitulées respectivement «contrôle du destin » et «hypothèses de recherche ». Un échantillon d'entrepreneurs francophones du Nouveau-Brunswick et du nord-est de l'Ontario est à la base de l'étude. Le plan de travail est le suivant: en premier lieu, nous passerons la revue de la littérature sur le contrôle du destin, ainsi que sur les variables retenues pour l'étude, suivie des hypothèses de recherche. La méthodologie utilisée sera ensuite décrite et nous terminerons avec la présentation des résultats. 
FIGURE 1

\section{Relations entre les dimensions de l'entreprisse}

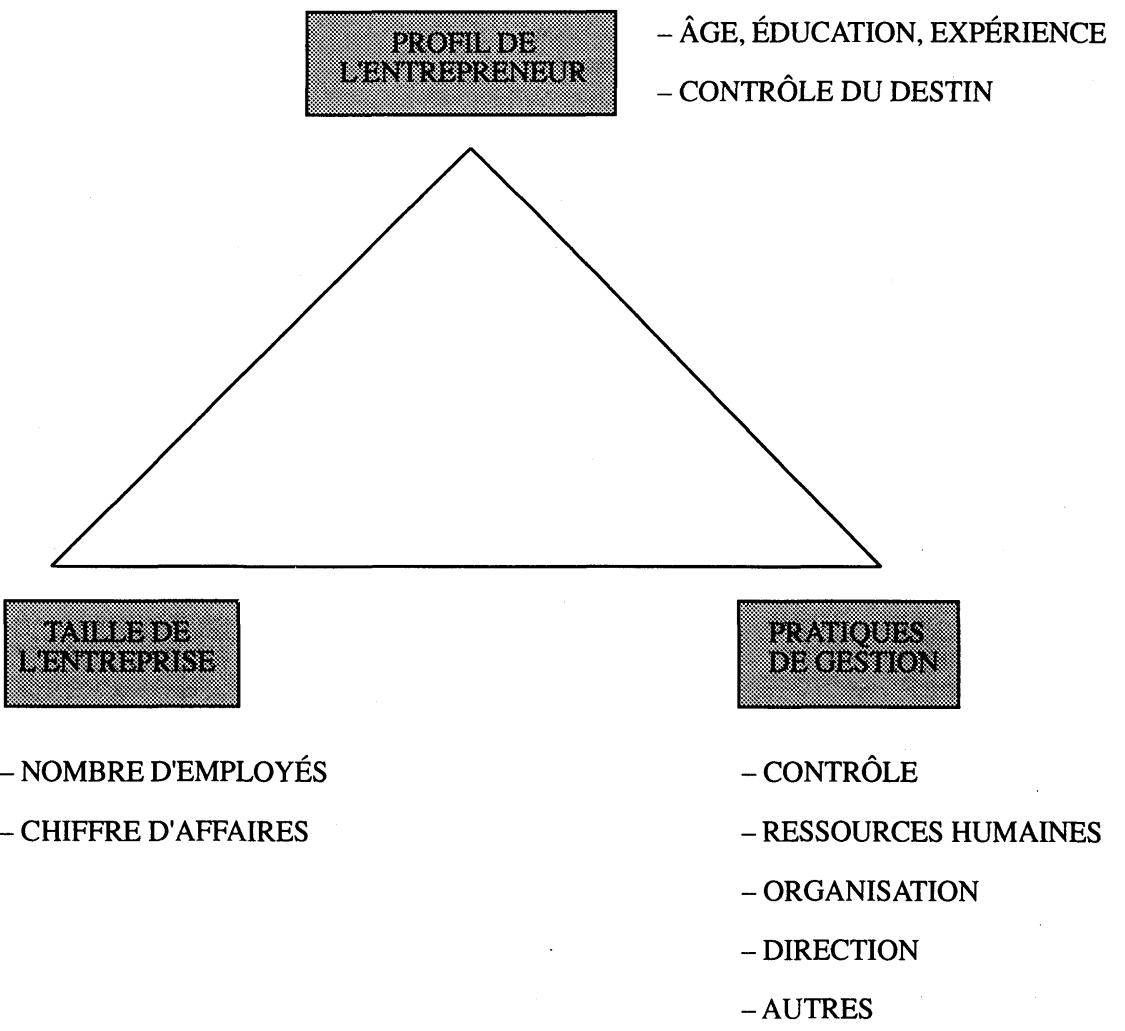

\section{Contrôle du destin}

Le concept de contrôle du destin tel que développé par Rotter en 1966, tout comme l'instrument qui le mesure (appelé échelle de Rotter), fait encore aujourd'hui l'objet d'une large utilisation en psychologie et dans d'autres domaines. Depuis, d'autres échelles de mesure ont été développées pour répondre aux besoins spécifiques de domaines tels que la santé physique et mentale, les croyances religieuses, l'éducation et le comportement humain à partir de l'enfance jusqu'à l'âge adulte (Furnham, 1986; Strickland, 1989).

Ce n'est que plus récemment que le concept du contrôle de destin fut appliqué au comportement organisationnel. Dans un essai de synthèse sur le comportement des organisations, Spector (1982) fait ressortir que les recherches antérieures ont établi une relation entre le concept de contrôle du destin chez 
l'employé, d'une part, et la motivation, l'effort, la performance, la satisfaction, la perception de son emploi, la conformité avec l'autorité et le style de supervision, d'autre part. Un fait intéressant au sujet de ces recherches est l'utilisation d'échelles généralisées pour mesurer l'internalité au lieu de modifier ou d'adapter ce concept aux caractéristiques particulières des domaines économiques ou organisationnels. Il est donc assez surprenant de constater que la majorité de ces chercheurs aient utilisé ou utilisent encore des échelles générales comme celle de Rotter (1966).

La littérature dans le contexte organisationnel mentionne également d'autres relations entre le trait de personnalité de contrôle du destin et d'autres variables reliées au domaine des affaires. Parmi celles-ci, les recherches de Miller et al. (1982) et Miller (1983) ont démontré que le degré de perception de contrôle des directeurs exécutifs de grandes entreprises tel qu'il a été mesuré par l'échelle de Rotter (1966) était fortement corrélé avec le degré d'innovation de l'entreprise, le niveau de planification et d'analyse effectué dans l'élaboration des stratégies et la nature de la structure organisationnelle. D'autres recherches établissant des liens entre le contrôle du destin et d'autres variables du domaine des affaires seront présentées plus loin dans le texte.

Dans un autre ordre d'idée, les résultats obtenus par Brockhaus (1975), Shapero (1975) et Brockhaus et Nord (1979) permettent d'établir que les entrepreneurs sont nettement plus «internes» que la population en général. Cependant, on ne dénote aucune différence significative entre les entrepreneurs et les gestionnaires, d'une part (Brockhaus et Nord, 1979; Mescon et Montanari, 1981 ; Sexton et Bowman, 1985), et les entrepreneurs fondateurs et non fondateurs de petites entreprises, d'autre part (Begley et Boyd, 1987). Toutefois, les recherches de Brockhaus (1975), Pandey et Tewary (1979) et de Cachon (1992) présentent des résultats contradictoires à ces dernières, les deux premières trouvant que les entrepreneurs sont plus internes que les managers, et celle de Cachon, que les entrepreneurs fondateurs sont plus internes que leurs confrères non fondateurs.

En dépit des nombreuses applications de l'échelle de contrôle du destin en management, il semble y en avoir très peu qui fassent le lien entre le contrôle du destin et les pratiques de gestion. Les seules recherches répertoriées en contexte canadien-français ont porté sur des échantillons assez modestes. Mentionnons ici celle de Lalonde et d'Amboise (1983) qui a porté sur un échantillon de 100 entreprises de la région du Saguenay-Lac-Saint-Jean et celle d'Amboise et Lalonde (1980) menée auprès d'un échantillon de 15 propriétaires-dirigeants œuvrant dans le domaine de l'industrie de la chaussure et des plastiques. 
À ce stade, nous pensons qu'il est important de préciser la définition attribuée dans notre étude au concept d'entrepreneur, éclaircissement opportun, étant donné l'ambiguïté reliée à la définition d'entrepreneur dans la littérature. La définition retenue est assez simple et d'ordre général et s'inspire de celle de Gasse et d'Amboise (1980) : c'est un individu qui participe dans une proportion importante au capital de l'entreprise et qui prend une part active aux décisions relatives à l'orientation de l'entreprise et à la solution des problèmes quotidiens de cette dernière. Cette définition comprend donc les individus qui fondent, achètent, héritent ou encore qui se joignent à l'entreprise après avoir acquis des actions, mais ne tient pas compte des gérants, lesquels ne participent pas au capital de l'entreprise. La définition retenue nous semble pertinente puisqu'elle met en évidence la notion de participation de l'entrepreneur à la conduite quotidienne des activités de son entreprise.

Les pages qui suivent présentent les hypothèses portant sur la relation entre certaines caractéristiques organisationnelles ou personnelles des dirigeants et les pratiques de gestion.

\section{Hypothèses de recherche}

Nos hypothèses de recherche s'inspirent, en partie, d'un modèle de recherche exploratoire développé initialement par Campbell et al. (1970) et utilisé par Lalonde et d'Amboise (1983). Ce modèle comporte un ensemble de variables regroupées autour de trois grandes dimensions de base, dont l'entreprise ellemême, l'entrepreneur et les pratiques de gestion. Ce modèle repose sur la notion que l'entrepreneur est en grande partie responsable de la croissance et du développement de son entreprise. Pour cette raison, notre définition d'entrepreneur semble appropriée, puisqu'elle met en évidence la notion de participation aux activités de l'entreprise.

C'est à l'aide de ce cadre général que furent testées les hypothèses dont il est question dans les sections qui suivent.

\subsection{Information générale}

La recherche en théorie des organisations tend à faire ressortir que l'utilisation des pratiques de gestion dépend en outre de la taille de l'organisation, laquelle est considérée, dans la littérature, comme l'un des principaux facteurs d'utilisation des pratiques de gestion (Lalonde, 1984). Ainsi, plus une entreprise comptera d'employés, plus elle sera susceptible de faire usage de pratiques de gestion. Les recherches de d'Amboise (1974), de Gasse et d'Amboise (1980) et de Gasse (1989) soutiennent également une telle relation. Dans cette dernière 
recherche, Gasse a trouvé une corrélation plus significative, entre la taille et les pratiques de gestion, au-delà de 30 employés.

À partir de ces recherches, nous proposons l'hypothèse suivante :

HYPOTHÈSE 1: Les pratiques de gestion varieront en fonction de la taille de l'entreprise

\subsection{Relation entre caractéristiques personnelles et pratiques de gestion}

La littérature rapporte un certain nombre de recherches sur la relation entre caractéristiques personnelles de l'entrepreneur et pratiques de gestion. Plus particulièrement, la relation entre le niveau et le type d'éducation reçus et l'expérience acquise contribuerait à une plus forte utilisation des pratiques de gestion (d'Amboise, 1974 ; Lalonde, 1984).

À partir de ces résultats, il nous apparaît intéressant de vérifier dans quelle mesure de telles relations peuvent exister au sein de notre échantillon d'entrepreneurs.

HYPOTHÈSE 2: Plus le niveau d'éducation de l'entrepreneur sera élevé, plus il utilisera des pratiques de gestion au sein de son entreprise.

HYPOTHĖSE 3: Cette relation sera plus forte lorsque l'entrepreneur aura acquis sa formation dans le domaine des affaires.

HYPOTHĖSE 4: Plus l'entrepreneur aura bénéficié d'une longue expérience de travail avant de prendre la direction de l'entreprise, plus il utilisera des pratiques de gestion.

HYPOTHÈSE 5: Cette relation sera plus forte dans le cas des entrepreneurs ayant acquis une expérience de direction.

\subsection{Relation attitude des entrepreneurs (contrôle du destin) et pratiques de gestion}

Comme nous l'avons mentionné précédemment, Phares (1968), Seeman (1967) et Seeman et Evans (1962) ont étudié le processus de collecte et d'utilisation d'information chez les individus dans leur tentative de minimiser l'incertitude. Ils ont conclu que la dimension contrôle du destin influençait à la hausse la recherche et l'utilisation d'information. Plus particulièrement, Davis et Phares (1967) ont démontré que les personnes internes recherchent plus activement de l'information qui est pertinente à la résolution de problèmes. 
Un tel comportement peut s'expliquer par le fait que les personnes qui présentent un concept de soi plus fort vers l'internalité sont plus convaincues que les renforcements peuvent provenir de leurs propres efforts. Quant aux personnes présentant un concept de soi vers l'externalité, ils pensent que leurs efforts ne sont pas importants dans l'atteinte d'un résultat ou d'une récompense. Nous pouvons donc supposer que les personnes ayant obtenu un résultat plus élevé d'internalité feront une utilisation plus active des pratiques de gestion, puisque celles-ci leur procureront l'information nécessaire à la résolution de problèmes.

Ce développement nous amène à proposer les hypothèses suivantes :

HYPOTHÈSE 6: Plus les entrepreneurs démontreront un niveau élevé d'internalité, plus ils feront usage des pratiques de gestion.

HYPOTHÈSE 7: Plus les entrepreneurs démontreront un niveau élevé d'internalité, plus ils feront usage de pratiques de contrôle.

D'autres études (Licata et al., 1986; Brownell, 1982) ont démontré que le trait de personnalité «contrôle du destin » avait une influence positive sur la consultation et la participation. L'étude de Licata et al. a révélé que les superviseurs classifiés «internes» consultaient plus que les superviseurs externes. De même, l'étude de Brownell démontrait que des employés classifiés «internes» participaient plus activement au processus budgétaire que leurs collègues classifiés «externes».

À partir de ces recherches, nous proposons l'hypothèse suivante:

HYPOTHÈSE 8: Plus les entrepreneurs démontreront un niveau élevé d'internalité, plus ils consulteront leurs subordonnés.

\section{Méthodologie}

\subsection{Instrument}

La méthodologie utilisée dans la présente étude reposait sur l'utilisation d'un questionnaire. Ce questionnaire fut prétesté auprès d'un échantillon de 16 entrepreneurs et des révisions mineures lui furent apportées à la lueur des commentaires reçus. Pour nous assurer d'un taux de retour satisfaisant, tous les répondants furent en premier lieu contactés par téléphone, pour savoir s'ils désiraient participer à l'étude. Si l'entrepreneur n'était pas disponible au moment de notre appel, celui-ci était contacté à nouveau un peu plus tard jusqu'à ce qu'il soit rejoint. Cette méthode permit l'envoi de 1317 questionnaires et fut suivie d'un rappel téléphonique pour s'informer auprès du répondant s'il avait reçu le 
questionnaire et, s'il y avait lieu, pour clarifier certains points des répondants. L'étude fut menée entre le 20 mai et le 15 juillet 1992.

Afin de faciliter l'analyse des résultats, les variables considérées dans le questionnaire furent regroupées autour des activités de direction, de contrôle, d'organisation et de gestion des ressources humaines. Le choix des pratiques de gestion s'appuie sur des recherches antérieures et prend en considération les restrictions imposées par le type d'outil utilisé pour recueillir les données. Bien entendu, ces variables ne sont pas les seules pouvant décrire de façon appropriée l'ensemble du processus de gestion. Le tableau 1 présente les pratiques de gestion utilisées représentant les activités de contrôle, de gestion des ressources humaines, d'organisation et de direction. Pour les activités de contrôle et de gestion des ressources humaines qui comprenaient plusieurs pratiques de gestion, des tests de fiabilité furent effectués. Les résultats de ces tests représentés par le coefficient alpha sont également présentés. Dans chacun des cas, les résultats obtenus ont excédé la valeur de 0,5 jugée acceptable par Nunnally (1970), confirmant la fiabilité des variables pour représenter les activités de gestion.

TABleau 1

Tableau des pratiques de gestion

\begin{tabular}{llc}
\hline $\begin{array}{c}\text { Activités de } \\
\text { gestion }\end{array}$ & \multicolumn{1}{c}{ Pratiques de gestion } & $\begin{array}{c}\text { Coefficient } \\
\text { alpha }\end{array}$ \\
\hline Contrôle & $\begin{array}{l}\text { - budget des ventes } \\
\text { - budget des dépenses d'opération } \\
\text { - budget de caisse } \\
\text { - contrôle de l'inventaire } \\
\text { - états financiers intérimaires }\end{array}$ & 0,86 \\
Gestion des ressources & - description écrite pour les tâches & \\
humaines (GRH) & - procédures écrites pour : & 0,83 \\
& - recrutement & \\
& - sélection & \\
& - entrânement & \\
Organisation & - évaluation & \\
Direction & - développement & s.o.* \\
Contrôle (autres) & - organigramme écrit & s.o.* \\
& - consultation des employés & s.o.* \\
& - états financiers prévisionnels & s.o.* \\
\hline
\end{tabular}

* Sans objet car une seule variable. 
L'échelle de Neal et Seeman (1964) fut utilisée pour mesurer le degré d'internalité obtenu des répondants. Cette échelle unidimensionnelle et constituée de sept énoncés présente un choix entre le sentiment de contrôle et d'impuissance face à des événements politiques, économiques et internationaux ${ }^{2}$. Un point était donné pour chaque réponse «externe». Plus le résultat obtenu sur l'échelle était élevé, plus le répondant était considéré externe (plus faible était sa perception de contrôle sur les événements présentés). À ce propos, notre échantillon s'est avéré être assez interne avec une moyenne de 2,69 sur une possibilité de 7 et un écart type de 1,75 .

Les relations entre les variables furent analysées au moyen du test de distribution chi-deux. Ce test, développé par Pearson, permet de calculer s'il y a effectivement une différence entre les pratiques de gestion et certaines caractéristiques personnelles (incluant le contrôle du destin) de deux ou plusieurs groupes d'individus.

Pour valider les résultats, nous avons effectué un test où un ensemble de variables identifiées comme des facteurs déterminants pour réussir en affaires furent regroupées. Ces quatre variables qui sous-entendaient soit l'internalité, soit l'externalité tentaient de mesurer la tendance interne ou externe des répondants. Cette échelle de mesure était constituée des énoncés suivants :

\section{Variables}

- avoir du personnel compétent

- se doter d'un bon système d'information

- avoir de la chance

- travailler de longues heures
Tendance

interne

interne

externe

externe

Cette échelle s'est révélée significative à un degré de 0,0075 .

\section{2. Échantillon}

La région du nord-est ontarien étudiée comprenait les entrepreneurs francophones des villes de Sudbury, North Bay, Timmins, Kapuskasing, Hearst, ainsi que les régions de Sudbury, Sturgeon Falls et Noelville. Étant donné le peu d'entreprises francophones, toutes celles dont le propriétaire était francophone et dont le nom apparaissait dans la troisième édition du répertoire des gens d'affaires francophones du nord-est ontarien, ainsi que sur les listes des

2. Le choix de l'échelle de Neal et Seeman fut motivé, d'une part, par les restrictions imposées pour contenir le questionnaire le plus court possible avec sept énoncés seulement et, d'autre part, par son utilisation très répandue (Cachon, 1988), ainsi que par la validité de l'échelle en elle-même (Seeman et Evans, 1962 ; Miller, 1976). 
membres des chambres de commerce, furent contactées. Pour le NouveauBrunswick, vu le nombre plus imposant d'entreprises francophones, l'échantillon fut formé aléatoirement à partir des entreprises dont le nom figurait dans les répertoires des Commissions industrielles francophones du NouveauBrunswick et de l'ensemble des entreprises du Conseil économique du Nouveau-Brunswick.

Des 1317 questionnaires initialement envoyés, 469 furent retournés, pour un taux de réponse de $35,6 \%$. Le tableau 2 présente l'échantillon des entreprises étudiées par secteur industriel et par province.

\section{TABleau 2}

Entreprises étudiées par secteur industriel et par province

\begin{tabular}{lccc}
\hline & $\begin{array}{c}\text { Ontario } \\
\mathbf{N = \mathbf { 2 0 9 }}\end{array}$ & $\begin{array}{c}\text { Nouveau-Brunswick } \\
\mathbf{N = \mathbf { 2 6 0 }}\end{array}$ & $\begin{array}{c}\text { Total } \\
\mathbf{N = \mathbf { 4 6 9 }}\end{array}$ \\
\hline $\begin{array}{l}\text { MANUFACTURIER } \\
\text { Total }\end{array}$ & $30,14 \%$ & $67,26 \%$ & 97 \\
$\begin{array}{l}\text { COMMERCE AU DÉTAIL } \\
\text { Total } \\
\text { SERVICES }\end{array}$ & $111,53 \%$ & $97,37 \%$ & 208 \\
Total & $68,33 \%$ & $96,37 \%$ & 164 \\
\hline
\end{tabular}

On retrouve dans l'échantillon du nord-est de l'Ontario une représentation plus élevée des secteurs de détail et de service par rapport au secteur manufacturier. Toutefois, ces résultats reflètent la composition de la population des affaires du nord-est ontarien, celle-ci étant surtout orientée vers ces secteurs; le secteur manufacturier consistant principalement en des activités de support aux quelques grandes entreprises manufacturières de la région. Par ailleurs, la proportion élevée des entreprises manufacturières de l'échantillon du Nouveau-Brunswick ( $26 \%$ ) peut s'expliquer par le fait que $62 \%$ des répondants étaient membres du Conseil économique du Nouveau-Brunswick, organisme élitiste qui n'est pas nécessairement représentatif de la population des affaires de cette province.

Le tableau 3 présente l'échantillon des entreprises par rapport à leur âge, ainsi que leur taille (nombre d'employés et chiffre d'affaires). Les résultats du tableau 3 portant sur la taille (nombre d'employés) nous démontrent que l'échantillon des entreprises est largement composé de petites entreprises. En effet, $49 \%$ des répondantes $(226 / 463)$ avaient 5 employés et moins ; $29 \%$, de 6 à 15 employés $(134 / 463) ; 22 \%, 16$ employés et plus (103/463). 
TABLEAU 3

Âge, taille et chiffre d'affaires

\begin{tabular}{|c|c|c|c|c|}
\hline VARIABLES & $\begin{array}{c}\text { Manufacturier } \\
\quad \mathbf{N}=97\end{array}$ & $\begin{array}{c}\text { Détail } \\
\mathbf{N}=\mathbf{2 0 8}\end{array}$ & $\begin{array}{l}\text { Service } \\
N=163\end{array}$ & $\begin{array}{c}\text { Total } \\
N=468\end{array}$ \\
\hline \multicolumn{5}{|l|}{$\begin{array}{l}\text { ÂGE } \\
\text { (nombre d'années) }\end{array}$} \\
\hline 1 à 5 ans & 14 & 39 & 40 & 93 \\
\hline 6 à 10 ans & 25 & 45 & 38 & 108 \\
\hline 11 à 20 ans & 35 & 63 & 39 & 137 \\
\hline $\begin{array}{l}20 \text { ans et }+ \\
\text { sans réponse }\end{array}$ & 0 & 3 & 1 & 4 \\
\hline \multicolumn{5}{|l|}{ TAILLE } \\
\hline 0 à 531 & 58 & 45 & 134 & \\
\hline 6 à 15 & 38 & 43 & 44 & 103 \\
\hline $\begin{array}{l}16 \text { et }+0 \\
\text { sans réponse }\end{array}$ & 3 & 2 & 5 & \\
\hline \multicolumn{5}{|l|}{ TAILLE } \\
\hline (chiffre d'affaires) & 13 & 46 & 72 & 131 \\
\hline $0-200000 \$$ & 22 & 40 & 33 & 95 \\
\hline $200000-500000 \$$ & 15 & 43 & 29 & 87 \\
\hline $500000-1 \mathrm{M} \$$ & 46 & 77 & 28 & 151 \\
\hline $\begin{array}{l}1 \mathrm{M} \$ \text { et }+ \\
\text { sans réponse }\end{array}$ & 1 & 2 & 1 & 4 \\
\hline
\end{tabular}

TABLEAU 4

Hypothèse 1

Taille vs pratiques de gestion

(nombre d'employés)

\begin{tabular}{cccccc}
\hline Organisation & RH & Direction & Contrôle & Prévision & Informatique \\
\hline 42,51 & 39,68 & 43,74 & 21,94 & 13,88 & 61,61 \\
$(0,0000)$ & $(0,0000)$ & $(0,0000)$ & $(0,00002)$ & $(0,00097)$ & $(0,0000)$ \\
\hline
\end{tabular}

\section{Résultats}

Les résultats obtenus pour l'hypothèse 1 appuient fortement l'existence d'une relation entre les pratiques de gestion et la taille, mesurée par le nombre d'employés $^{3}$ (tableau 4). En effet, un degré de signification de 0,0000 a été obtenu

3. En utilisant le chiffre d'affaires pour mesurer la taille, les résultats obtenus se sont révélés significatifs à un degré équivalent. 
pour les activités de gestion des ressources humaines, d'organisation (organigramme écrit) et d'informatique. Les activités de contrôle étaient significatives à un seuil de 0,00002 et l'utilisation d'états financiers prévisionnels, de 0,00097 , confirmant une relation évidente entre l'utilisation de pratiques de gestion et la taille de l'entreprise.

L'hypothèse 2 portait sur la relation entre le niveau d'éducation et les pratiques de gestion. De façon générale, les résultats ont démontré qu'il y avait une différence significative entre le niveau d'éducation (secondaire, collégial ou universitaire) et les pratiques de gestion (tableau 5). L'hypothèse 2 , à l'exception du contrôle (degré de signification de 0,17 ) est nettement supportée, les répondants ayant fait des études universitaires ou collégiales faisaient en général un plus grand usage des pratiques de gestion que ceux qui avaient une formation secondaire et moins. Ces résultats sont confirmés aux tableaux 5a, $5 \mathrm{~b}$ et $5 \mathrm{c}$ qui présentent les résultats comparatifs pour chacun des deux niveaux d'éducation.

TABLEAU 5

Hypothèse 2

Éducation vs pratiques de gestion

(Études secondaires vs collège vs université)

\begin{tabular}{cccccc}
\hline Organisation & RH & Direction & Contrôle & Prévision & Informatique \\
\hline 22,81 & 28,91 & 25,93 & 3,53 & 10,68 & 50,67 \\
$(0,00001)$ & $(0,00001)$ & $(0,0000)$ & $(0,17)$ & $(0,00497)$ & $(0,0000)$ \\
\hline
\end{tabular}

TABleau 5a

Hypothèse 2a

(Études secondaires vs collège vs université)

\begin{tabular}{cccccc}
\hline Organisation & RH & Direction & Contrôle & Prévision & Informatique \\
\hline 3,02 & 15,55 & 2,65 & 0,388 & 2,36 & 3,32 \\
$(0,082)$ & $(0,0004)$ & $(0,103)$ & $(0,533)$ & $(0,124)$ & $(0,068)$ \\
\hline
\end{tabular}

TABLEAu $5 b$

Hypothèse $2 b$

(Études secondaires vs université)

\begin{tabular}{cccccc}
\hline Organisation & RH & Direction & Contrôle & Prévision & Informatique \\
\hline 4,40 & 4,76 & 6,61 & 0,791 & 1,14 & 12,16 \\
$(0,036)$ & $(0,092)$ & $(0,01)$ & $(0,374)$ & $(0,285)$ & $(0,0005)$ \\
\hline
\end{tabular}




\section{TABleau 5c}

Hypothèse 2c

(Études secondaires vs université)

\begin{tabular}{cccccc}
\hline Organisation & RH & Direction & Contrôle & Prévision & Informatique \\
\hline 22,83 & 19,77 & 25,98 & 3,53 & 10,39 & 39,84 \\
$(0,00000)$ & $(0,00005)$ & $(0,00000)$ & $(0,060)$ & $(0,0013)$ & $(0,0000)$ \\
\hline
\end{tabular}

Les résultats du tableau 5a (hypothèse $2 a$ ) démontrent qu'il y a peu de différence entre les pratiques de gestion utilisées par les répondants qui détiennent un diplôme d'études secondaires de ceux qui ont terminé leurs études collégiales. Seulement une fonction était significative $(\mathrm{RH}, \mathrm{p}=0,00004)$. La différence est un peu plus significative entre des études universitaires et des études collégiales où l'on dénote trois fonctions significativement différentes (organisation, $\mathrm{p}=0,036$, direction, $\mathrm{p}=0,01$, et informatique, $\mathrm{p}=0,0005$ ) (tableau 5b, hypothèse $2 \mathrm{~b}$ ). Par contre, la différence est nettement plus significative si on compare les répondants avec une formation secondaire avec les répondants détenant une formation universitaire (tableau $5 \mathrm{c}$, hypothèse $2 \mathrm{c}$ ). Il existe une différence significative pour presque toutes les pratiques de gestion et le degré de signification pour les pratiques de contrôle passe de 0,17 à 0,06 .

L'hypothèse 3 établissait une relation entre le type d'éducation et les pratiques de gestion. Les résultats du tableau 6 ne supportent pas l'hypothèse, puisque aucune différence significative n'a été observée dans les pratiques de gestion entre les répondants détenant une formation postsecondaire (collégiale et universitaire) ayant reçu une éducation dans un programme de gestion et une formation autre qu'en affaires. Les résultats semblent indiquer qu'une plus grande utilisation des pratiques de gestion est liée à l'éducation postsecondaire, quel que soit le domaine de formation. Ces derniers résultats confirment les résultats obtenus par d'Amboise (1974) et Lalonde (1984) à ce sujet.

Tableau 6

Hypothèse 3

Type d'éducation vs pratiques de gestion

(Formation en affaire vs autre)

\begin{tabular}{cccccc}
\hline Organisation & RH & Direction & Contrôle & Prévision & Informatique \\
\hline 0,024 & 0,453 & 1,256 & 0,0026 & 1,456 & 1,487 \\
$(0,878)$ & $(0,797)$ & $(0,262)$ & $(0,959)$ & $(0,2275)$ & $(0,476)$ \\
\hline
\end{tabular}

Finalement, les résultats obtenus pour l'expérience font ressortir que l'expérience en gestion acquise précédemment a un impact positif sur l'utilisation des pratiques de gestion (hypothèse 5 , tableau 7) comparativement 
à l'expérience en général dont les différences étaient moins significatives (hypothèse 4, tableau 6). Ces résultats se justifient, puisqu' une personne ayant déjà été en contact avec de telles pratiques aura tendance à les utiliser à son tour lorsque l'occasion se présentera.

TABLEAU 7

Hypothèse 4

Expérience vs pratiques de gestion (Général)

\begin{tabular}{cccccc}
\hline Organisation & RH & Direction & Contrôle & Prévision & Informatique \\
\hline 1,912 & 7,60 & 3,355 & 3,18 & 4,911 & 0,409 \\
$(0,166)$ & $(0,022)$ & $(0,067)$ & $(0,074)$ & $(0,0267)$ & $(0,815)$ \\
\hline
\end{tabular}

TABLEAU 7

Hypothèse 5

Expérience vs pratiques de gestion (Expérience en gestion)

\begin{tabular}{cccccc}
\hline Organisation & RH & Direction & Contrôle & Prévision & Informatique \\
\hline 3,812 & 12,84 & 4,135 & 12,94 & 8,48 & 1,97 \\
$(0,050)$ & $(0,0016)$ & $(0,042)$ & $(0,0005)$ & $(0,0036)$ & $(0,373)$ \\
\hline
\end{tabular}

TABLEAu8

Hypothèse 6

Contrôle du destin vs pratiques de gestion (interne $(0,1)$ vs externe $(5,6,7)$

\begin{tabular}{cccccc}
\hline Organisation & RH & Direction & Contrôle & Prévision & Informatique \\
\hline 6,12 & 4,87 & 12,43 & 5,27 & 3,06 & 4,97 \\
$(0,013)$ & $(0,087)$ & $(0,0004)$ & $(0,022)$ & $(0,080)$ & $(0,026)$ \\
\hline
\end{tabular}

Pour les hypothèses 6,7 et 8 , les résultats des pratiques de gestion furent comparés avec les répondants classifiés internes et externes selon l'échelle de Neal et Seeman. Pour déterminer les groupes d'individus internes et externes, nous avons retenu les répondants dont le score était supérieur ou inférieur de un écart type de la moyenne. Les résultats ainsi obtenus nous ont permis de procéder aux analyses. À l'exception des pratiques reliées à la gestion des ressources humaines $(0,087)$, toutes les autres pratiques se sont révélées significatives, comme le démontrent les résultats du tableau 8 . Les pratiques de gestion telles que l'organisation (organigramme écrit, $p=0,013$ ), la direction (consultation avec les subordonnés, $p=0,0004)$, le contrôle $(p=0,022)$, 
et l'utilisation de l'informatique $(\mathrm{p}=0,026)$ étaient toutes significatives. Cela confirme donc nos hypothèses selon lesquelles le contrôle du destin est relié chez les entrepreneurs à une plus grande utilisation des pratiques de gestion et à une plus grande consultation des subordonnés.

\section{Conclusion}

La présente recherche avait pour objet d'étudier la relation entre certaines caractéristiques de l'entreprise et des entrepreneurs sur les pratiques de gestion. Les résultats ont démontré que certaines variables telles que la taille de l'entreprise, le type, ainsi que le niveau d'éducation ont un effet sur les pratiques de gestion. En effet, plus le niveau de scolarisation est élevé, plus on pratique des activités de gestion. Des résultats similaires furent observés avec la taille de l'entreprise. D'autres variables mesurant les caractéristiques personnelles des dirigeants se sont aussi révélées significatives. La variable contrôle du destin fut reliée à une plus grande utilisation des pratiques de gestion par les entrepreneurs. Les résultats corroborent la plupart des hypothèses avancées ainsi que la littérature à ce sujet. La taille de notre échantillon, ainsi que la rigueur des tests statistiques utilisés, nous ont permis d'obtenir des résultats intéressants.

L'intérêt de cette recherche est qu'elle permet d'avoir une meilleure compréhension des facteurs qui influencent l'utilisation des pratiques de gestion. Il devient ainsi possible de développer un profil des entrepreneurs qui font un plus grand usage des pratiques de gestion et de créer un modèle permettant de déterminer qui serait susceptible de mieux réussir en affaires. Les études futures devraient tenir compte de l'intégration entre les variables et essayer d'identifier la ou les variables les plus importantes dans l'utilisation des pratiques de gestion.

\section{Bibliographie}

BEGLEY, T. et D. BOYD (1987), «Psychological characteristics associated with performance in entrepreneurial firms and smaller businesses », Journal of Small Venturing, no 2, p. 79-93.

BORLAND, C. (1975), «Locus of control, need for achievement and entrepreneurship », Thèse de doctorat, Austin, University of Texas.

BOyD, D. et D. GuMPERT (1984), «The loneliness of the start up entrepreneurs », Communication à la conférence de Babson sur l'entrepreneur, Atlanta, Georgia Tech University. 
BRoCKHAUS, R.H. (1975), «I-E locus of control scores preditors of entrepreneurial intentions », Comptes rendus, New Orleans, Academy of Management.

BROCKHAUS, R.H. et W.R. NORD (1979), «An exploration of the factors affecting entrepreneurial decision : personal characteristics vs. environmental conditions », Comptes rendus, Atlanta, Academy of Management, p. 364-368.

BROWNELl, P. (1982), «A field study of budgetary participation and locus of control », Accounting Review, vol. 57, n 4, p. 766-777.

CACHON, J.C. (1988), «Venture creators and firms buyers : a comparison of attitudes towards government help and locus of control», dans A. Bruce Kirchoff (éd.), Frontiers of Entrepreneurship Research, Wellesley, Babson College, chap. 31, p. 568-579.

CACHON, J.C. (1992), «Entrepreneurs : pourquoi ? comment ? quoi ?», Revue du Nouvel-Ontario, $\mathrm{n}^{\text {os }} 13-14$, p. 13-56.

Campbell, J.P., M.D. Dunette, E.E. Lawler et K.E. Weick (1970), Managerial Behavior, Performance and Effectiveness, New York, McGraw-Hill.

D’AMBoise, G.R. (1974), «Personal characteristics, organizational practices and managerial effectiveness : a comparative study of French and English speaking chief executives in Québec », Thèse de doctorat, Los Angeles, University of California.

D'Amboise, G. et C. LALONDE (1980), «Faillites d'entreprises : une étude préliminaire», Document de travail, Université du Québec à Chicoutimi.

DAVIS, W.L. et E.J. PHARES (1967), « Internal-External as a determinant of informationseeking in a social influence situation », Journal Personality, $\mathrm{n}^{\circ} 35$, p. 547-561.

FURnhaM, A. (1986), «Economic locus of control », Human Relations, vol. 39, $\mathrm{n}^{\circ} 1$, p. 29-43.

GASSE, Y. et G. D'AMBOISE (1980), «Identification des difficultés managériales et des facteurs de succès dans les PME québécoises : une proposition de recherche », Cahier de recherche 80-34, Faculté des sciences de l'administration, Université Laval.

GASSE, Y. (1989), «L'utilisation de diverses techniques et pratiques de gestion dans les PME», Revue PMO, vol. 4, nº 1, p. 3-11.

Gordon, L.A. et D. MiLleR (1978), «A contingency framework for the design of accounting information systems », dans R. Hector Anton, A. Peter Firmin et Hugh Grove (éd.), Contemporary Issues in Cost and Management Accounting, Boston, Houghton Mifflin Company, p. 516-517.

HENDRIX, J.A. (1985), «Locus of control: implications for managers and accountants », Cost and Management, vol. 39, mai-juin, p. 25-29. 
HORNADAY, J.A. et J. ABOUD (1971), «Characteristics of successful entrepreneurs », Personnel Psychology, $\mathrm{n}^{\circ} 24$, p. 141-153.

KETS DE VRIES, M.F.R. (1977), «The entrepreneurial personality: a person at the crossroads », Journal of Management Studies, vol. 14, p. 34-57.

LALONDE, C. et G. D'AMBoISE (1983), «Le propriétaire-dirigeant failli : les éléments d'un profil», Revue PMO, p. 5-19.

LALONDE, C. (1984), «Faillites d'entreprises : une étude en contexte régional au Québec », Thèse de doctorat, Université Laval.

LeS PROMOTIONS NoRdCom INC. (1992-1993), Répertoire des gens d'affaires francophones du Nord-Est de l'Ontario, $4^{\mathfrak{c}}$ édition.

LiCATA, M.P., R.H. STAWSER et R.B. WELKER (1986), «A note on participation in budgeting and locus of control », Accounting Review, vol. 61, p. 112-118.

LITZINGER, W.D. (1965), "The motel entrepreneur and the motel manager», Academy of Management Journal, n 8, p. 793.

MESCON, T.S. et J.R. MONTANARI (1981), «The personalities of independant and franchise entrepreneurs », Comptes rendus, Academy of Management, p. 413-417.

Miller, D. (1976), Handbook of Research Design and Social Measurement, New York, David McKay Company.

Miller, D., M.F.R. KetS DE VRIES et J.M. Toulouse (1982), « Top executive locus of control and its relationship to strategy-making, structure and environment », Academy of Management Journal, vol. 25, $\mathrm{n}^{\circ}$ 2, p. 237-253.

MilleR, D. (1983), "The correlates of entrepreneurs in three types of firms", Management Science, vol. 29, $\mathrm{n}^{\circ}$ 7, p. 770-791.

Miller, D. et J.M. Toulouse (1986), « Chief executive personality and corporate strategy and structure in small firms", Management Science, vol. 32, novembre, p. 1368.

Neal, A.G. et M. Seeman (1964), "Organization and powerlessness : a test of the mediation hypothesis », American Sociological Review, n² 29, p. 216-226.

Nunnally, J. (1970), Introduction to Psychological Measurement, New York, McGraw-Hill.

PANDEY, J. et N.B. TEWARY (1979), «Locus of control and achievement values of entrepreneurs », Journal of Occupational Psychology, vol. 52, n 2, p. 107-111.

PHARES, E.J. (1968), « Differential utilization of information as a function of internalexternal control », Journal of Personality, n 36, p. 649-662.

RoTTER, J.B. (1966), «Generalized expectancies for internal versus external control of reinforcement : a case history of a variable », Psychological Monographs, vol. $80, \mathrm{n}^{\circ} 1$, p. $260-295$. 
SeEman, M. et J.W. Evans (1962), «Alienation and learning in a hospital setting », American Sociological Review, $\mathrm{n}^{\circ} 27$, p. 772-793.

Seeman, M. (1967), "On the personal consequences of alienation in work», American Sociological Review, $\mathrm{n}^{\circ}$ 32, p. 973-977.

SEXTON, D.L. et N. Bowman (1985), «The entrepreneur: a capable executive and more », Journal of Small Venturing, $\mathrm{n}^{\circ} 1$, p. 129-140.

Shapero, A. (1975), «The displaced, uncomfortable entrepreneur », Psychology Today, vol. 11, n 7 , p. 83-86.

SPECTOR, P. (1982), « Behavior in organizations as a function of employees' locus of control », Psychological Bulletin, n 91 , p. 482-497.

STRICKLAND, B.R. (1989), «Internal-External control expectancies: from contingency to creativity », American Psychologist, n 44, p. 1-12. 